\title{
Metrical Tone Shift and Spread in Harmonic Serialism*
}

\author{
Jeroen Breteler \\ University of Amsterdam
}

\section{Introduction}

Some Bantu languages allow tone to spread or shift, but only within a limited domain. This domain is typically two or three syllables in size. A simplified overview of attested patternsis shown in Table 1.

\begin{tabular}{|c|c|c|c|}
\hline Pattern & $\mathbf{U F}$ & SF & Example attestation \\
\hline Binary spreading & $. . \dot{\sigma} \sigma .$. & $. . \dot{\sigma} \sigma .$. & Ekegusii (Bickmore, 1996) \\
\hline Ternary spreading &..$\sigma \sigma \sigma \sigma$. & ..óóó.. & Copperbelt Bemba (Bickmore \& Kula, 2013) \\
\hline Binary shift & $. . \dot{\sigma} \sigma .$. &..$\sigma \sigma_{. .}$ & Kikuyu (Clements, 1984) \\
\hline Bin. shift + bin. spread &..$\sigma \sigma \sigma \sigma$. & 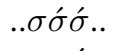 & Saghala (Patin, 2009) \\
\hline Ternary shift &..$\sigma \sigma \sigma \sigma$. & $. . \sigma \sigma \sigma ́ .$. & Sukuma (Sietsema, 1989) \\
\hline
\end{tabular}

Table 1: Bounded tone patterns.

Previous research has analysed bounded tone using foot structure (see Sietsema 1989; Bickmore 1996 for overviews). The core of such proposals is that metrical constituents delimit the domain of tonal mobility. However, such approaches run into problems for the analysis of ternary domains, and in implementing bounded tone shift in Optimality Theory (“OT”, Prince \& Smolensky, 1993). Consequently, OT approaches to bounded tone have explored other analytical means.

This paper returns to the foot-based analysis in the light of a reevaluation of metrical structure by Martínez-Paricio (2013); Martínez-Paricio \& Kager (forthcoming) et seq., hereafter "MPK". MPK propose a layered, maximally trisyllabic foot. This provides a natural way of capturing ternary tone phenomena. To account for tone shift in an OT context, the present analysis is couched in the Harmonic Serialism framework (McCarthy, 2010). The foot-based analysis is demonstrated here for Saghala (Patin, 2009), which features a pattern of shifting and spreading across a trisyllabic domain.

The next section discusses previous OT approaches to bounded tone and identifies two areas for improvement. Section 3 sets up the analytical framework, motivating the present constraint set and the adoption of Harmonic Serialism. Section 4 then applies the metrical approach to Saghala. Section 5 concludes the paper.

\section{Previous OT approaches to Bantu bounded tone}

Within the context of OT, three lines of previous research on Bantu bounded tone can be recognized: Optimal Domains Theory, minimal (mis)alignment, and headed spans theory.

Optimal Domains Theory ("ODT") centers around the idea of relating underlying tones to surface-level tone 'domains' (Kisseberth, 1993; Cole \& Kisseberth, 1994; Cassimjee \& Kisseberth, 1998). Bounded tone patterns then follow from restrictions on the size of such tone domains.

Bickmore's approach of minimal (mis)alignment derives surface tone patterns from a family of alignment constraints, which may cause tone to spread to tone bearing units ("TBUs") at a minimal distance away from

\footnotetext{
* Correspondence to j.m.w.breteler@uva.nl. I thank Paul Boersma and René Kager for supervision and many discussions of this work. Thanks to Cédric Patin for discussion on the data, as well as providing further examples and original recordings. I have also benefited from discussing this work with Lee Bickmore, as well as audiences of the lab meetings at U Amsterdam, the PhonoLAM meetings at U Amsterdam/Meertens Institute, the Sound Seminar at UMass Amherst, the Phonology 2014 conference at MIT, and the 12th Old World Conference of Phonology at U Autònoma de Barcelona and U Barcelona. Special thanks to my father for hearing me out when I got stuck. I am solely responsible for any errors.
}

(C) 2015 Jeroen Breteler

Proceedings of AMP 2014

Completed January 22, 2015 
their sponsor (Bickmore, 1996). The minimal distance effect is due to the gradient interpretation of the alignment constraints.

Headed spans theory proposes that surface forms are parsed exhaustively into domains for each feature ('feature spans'), notably tone (Key 2007; Key \& Bickmore 2014, building on McCarthy 2004). Much like in ODT, bounded patterns are derived by placing requirements on the size of such feature spans.

The above proposals suffer from either or both of the following problems: stipulation of domain size and use of two-level constraints. These issues are discussed in the following subsections.

2.1 Stipulation of domain size One of the goals for any account of Bantu bounded tone is to derive tonal spans over multiple TBUs starting from a tone with only a single underlying association. To this end, ODT employs a *MONOHD constraint to enforce binary domains. Likewise, in the Headed Spans framework, binarity is achieved through $\operatorname{SPBIN}(\mathrm{T})$. Hence, in both frameworks the impetus for binarity is stipulated; it does not follow from the theory of the representation.

Furthermore, neither framework has a way of accounting for ternary domains. Ternarity could be achieved by adding constraints such as *BINHD for ODT or SPTRI(T) for Headed Spans, but this adds further stipulations. Furthermore, there is no account for the fact that there are presumably no constraints such as *TRIHD or SPQUAD(T) that could drive construction of quaternary tonal spans.

In the present approach, binarity and ternarity are linked to the nature of the foot, the size of which is motivated independently. A quaternary domain cannot be derived straightforwardly, matching the typological picture of Bantu bounded tone.

2.2 Two-level constraints Two-level constraints enforce a relationship between input and output representations beyond simple correspondence. In the analysis of Bantu bounded tone, two-level constraints occur when constraints make reference to a 'sponsor'. Sponsorship is a property of a TBU at the underlying level of representation. Making surface requirements on sponsors means that both levels of representation are involved. ODT and the minimal (mis)alignment approach make use of exactly these kinds of constraints.

Two-level constraints go against a core principle of OT: its surface-orientedness. Consider the following criticism on these constraints from Kager (1999):

" [Two-level constraints] function as rules, combining a structural condition (the input structure) and a repair. A theory allowing for two-level well-formedness constraints may stipulate any type of relation between the input and output, being equivalent in this respect to rule-based theory (Lakoff 1993). This power undermines standard OT's solutions to problems inherent to rulebased serialism, in particular conspiracies and the Duplication Problem." (p. 381)

In conclusion, it is desirable to avoid the use of two-level constraints. This is achieved by the present proposal, as detailed in the next section.

\section{The metrical approach in Harmonic Serialism}

This section will outline a metrical approach to Bantu bounded tone in an OT context. First, the following subsection will discuss the metrical representations assumed here. Then, section 3.2 details the constraint set required to relate tone and feet. Section 3.3 will show that bounded tone shift cannot be derived with metrical structure in standard OT. Finally, section 3.4 discusses the adoption of Harmonic Serialism for the present purpose.

3.1 Layered Feet Following MPK (who follow Selkirk (1980); Prince (1980); Kager (1994)), we assume that metrical feet may be layered. That is, a binary foot may combine with a satellite syllable to form a layered, trisyllabic foot. The different foot layers are distinguished in terms of (non)minimality and maximality. Sample feet are shown in figure 1.

3.2 Constraints To regulate the relationship between tone and feet, two types of constraints are used: licensing and structural constraints.

Licensing constraints drive the association between tones and feet. Crucially, licensing constraints can take either the tone or the foot as the locus of violation. Two example definitions of licensing constraints follow below. 


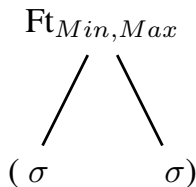

(a) A flat, binary foot.

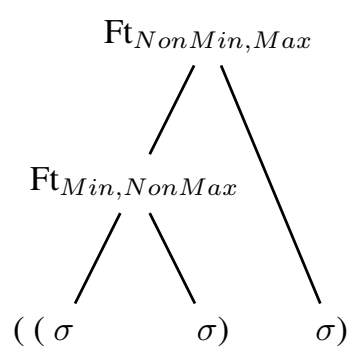

(b) A layered, ternary foot.

Figure 1: Binary and ternary foot types in the MPK framework.

LICENSE-H For each $\mathrm{H}$, assign * if it is not associated to a footed syllable.

LICENSE-FT For each foot, assign * if none of its syllables is associated to a $\mathrm{H}$
tone.

Depending on the number of feet and tones in a candidate form, the two constraints above may come out to different amounts of violation marks. For example, the constraint LICENSE-FT does not punish forms with stray $\mathrm{H}$ tones, as long as every foot in the form contains at least one high-toned syllable, unlike LICENSEH. Another important difference between the constraints is the means by which their violations may be avoided. For example, LICENSE-H could drive the deletion of tones that are not associated to a footed syllable. LICENSE-FT, on the other hand, is never helped by tone deletion, but instead this constraint might prevent the creation of unlicensed feet.

Structural constraints militate against an association between a $\mathrm{H}$ tone and a foot. For example:

\begin{tabular}{ll}
\hline$* \mathrm{H} / \mathrm{FT}$ & $\begin{array}{l}\text { Assign one } * \text { for each association between a } \mathrm{H} \text { tone and a footed } \\
\text { syllable. }\end{array}$ \\
\hline
\end{tabular}

In the case of structural constraints, the locus of violation is the association itself. Consequently, there is no need for a distinction between a tone-version and a foot-version of the structural constraints.

The above definitions are the general forms of the proposed constraints. The full power of the constraint set is activated by instantiating these constraints for specific layers of the foot and specific edges of feet. For example, the following three constraints show instantiations of these constraints for the right edges of minimal feet ("MinFt"):

$\begin{array}{ll}\text { LICENSE(H,MinFT- } & \begin{array}{l}\text { For each } \mathrm{H} \text {, assign * if it is not associated to a syllable that is } \\ \text { rightmost in a MinFt. }\end{array}\end{array}$

\begin{tabular}{ll}
\hline $\begin{array}{l}\text { LICENSE(MINFT- } \\
\mathrm{R}, \mathrm{H})\end{array}$ & $\begin{array}{l}\text { For each MinFt, assign * if its rightmost syllable is not associated to } \\
\text { a } \mathrm{H} \text { tone. }\end{array}$ \\
\hline$* \mathrm{H} /$ MINFT-R & $\begin{array}{l}\text { Assign one * for each association between a H tone and a syllable } \\
\text { that is rightmost in a MinFt. }\end{array}$ \\
\hline
\end{tabular}

Aside from licensing and structural constraints, some further constraints are adopted:

1. Foot directionality constraints. Specifically, $\operatorname{CHAIN}-\mathrm{L}\left(\sigma_{\omega}\right)$ and $\operatorname{CHAIN}-\mathrm{R}\left(\sigma_{\omega}\right)$, taken from MPK. These constraints are highly similar in function to ALL-FT-R/L, respectively. 
2. Conventional faithfulness constraints, i.e. MAX, DEP, UNIFORMITY, etc. for tones and association links.

3. NoFLOAT (McCarthy et al., 2012) to militate against floating tones.

3.3 The problem of metrical tone shift in Standard OT Standard OT is faced with a problem when accounting for metrical tone shift; it is never optimal to shift locally, i.e. in a bounded fashion. Consider a toy example where the aim is to do binary rightward shift. That is, an underlyingly linked tone should surface on the syllable following its sponsor. This toy analysis of shifting will involve the following effects:

1. Faithfulness; here, a general FAITH-T that is violated when the tone shifts.

2. Foot creation; LICENSE-H drives foot creation in places where there is tone.

3. Foot directionality; CHAIN-L $\left(\sigma_{\omega}\right)$ pulls unparsed syllables to the left, and consequently feet to the right.

4. Tone mobility; a specific licensing constraint, LiCEnSE(FT-R, H), drives tones to the right edges of feet.

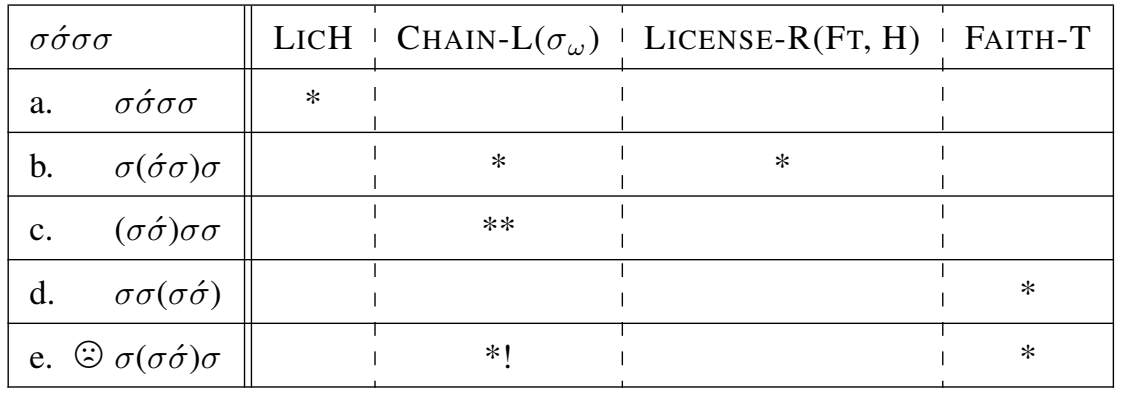

Tableau 2: Toy example of binary rightward shift in standard OT.

In Tableau 2, only candidates with a singly linked tone are considered. Note that while a number of candidates could be optimal for different rankings, the desired candidate $2 \mathrm{e}$, a local, one-syllable shift to the right, is harmonically bounded by candidate $2 \mathrm{~d}$. In conclusion, standard OT lacks a way of deriving the local shift effect. This problem can be solved by adopting the Harmonic Serialism framework, which is the topic of the next subsection.

3.4 Harmonic Serialism Harmonic Serialism ("HS") changes standard OT in two ways. Firstly, the generation of candidates is limited. Specifically, candidates can only differ from the input form by the application of one operation, defined below. Secondly, an evaluation happens serially, with the output of one tableau functioning as the input of the next, until no changes occur. These factors combined lead to a derivation consisting of local improvements.

For the present purposes, an operation can be the deletion or insertion of a tonal association link, or the construction of a foot. Some other plausible operations, such as inserting or deleting a tone, are not considered. These operations can be blocked by high-ranking faithfulness constraints.

Another operation to be considered is a tone shift operation, i.e. one that shifts a tone from its associated TBU to the next TBU. Such an operation is a composition of two other constraints already said to be in GEN: linking and delinking a tone. Consequently, for the sake of parsimony, the tonal shift operation is assumed to be excluded from GEN. ${ }^{1}$

In standard OT, locality had to be derived by making reference to tone sponsors, thus giving rise to twolevel constraint formulations. In HS, changes to the underlying form are serial. This means foot placement can make reference to the underlying location of tone as long as the derivation has not reached a step where

1 A decomposition of tone shift into linking and delinking is also in line with the findings of Odden (2001), although those conclusions were made outside of the context of HS. 
tone moves. Consequently, there is no need to explicitly refer to the concept of a tone sponsor anymore, and no need to use two-level constraints. At each step of the serial evaluation, markedness constraints are output-oriented and need not make reference to input-level structure.

Some other multi-level frameworks, such as Stratal OT (Kiparsky, 2000) and cophonology theory (Inkelas \& Zoll 2007 and references therein), are tied to morphological levels. This is not the case for HS. For the present purposes, this is fitting, as the tonology of Saghala takes place exclusively at the phrasal level.

\section{Case: Saghala}

All data is taken from reports by Patin $(2002,2009)$ on the tonology of noun phrases in Saghala (Guthrie's E74b), spoken in southeastern Kenya. The tonology is sensitive to the position of word boundaries and the proximity of tones to each other. However, this paper restricts itself to discussing the default case, i.e. the behavior of tones in contexts with only a single tone and no influence of word boundaries. For a full analysis of the Saghala data in the present framework, see Breteler (forthcoming).

The data in (1) demonstrate some of the basic facts of Saghala tone. Sponsors are marked by underlining, while surface-level highs are indicated with accents.
a. $\varnothing$ njovu 'elephant(s)'
b. izí nfóvu 'that elephant'
c. ilya nfóvú 'these elephants'

Firstly, note the tone shifting nature of the language: the bare noun in (1a) is toneless, but tone can be contributed to the noun from preceding determiners in $(1 \mathrm{~b}, \mathrm{c})$. Secondly, the determiners differ in terms of onset of the tonal span and, consequently, the degree to which the span crosses into the next word. This demonstrates that tone in Saghala is linked underlyingly, and can be linked to different places in a word. Finally, the data demonstrate the default pattern in Saghala: the two syllables following a sponsor receive high tone, while the sponsor itself is low-toned at the surface. The next section will account for this pattern formally.

4.1 Analysis The analysis of the Saghala default pattern will adopt the following constraint ranking:

1. MAX-T, DEP-T, NOFLOAT

Assign one * for each tone that is not associated to any TBU.

2. LICENSE-H (abbreviated LICH)

Assign one * for each $\mathrm{H}$ that is not associated to a footed syllable.

3. LICENSE-FT (abbreviated LICFT)

Assign one * for each foot if none of its syllables are associated to a $\mathrm{H}$ tone.

4. Chain-L $\left(\sigma_{\omega}\right)$ (abbreviated Chain-L)

Assign one * for each unparsed syllable that is not in a chain, i.e. unbroken sequence, of unparsed syllables starting from the left edge of the prosodic domain.

5. *H/MinFT-L (abbreviated *H/Min-L)

Assign one * for each association between a $\mathrm{H}$ and a syllable that is leftmost in a Min foot.

6. LiCEnse(H,MinFT-R) (abbreviated R(H,Min))

Assign one * for each $\mathrm{H}$ tone that is not associated to the rightmost syllable of a Min foot.

7. LiCEnSE(H,NonMin-R) (abbreviated R(H,NonMin))

Assign one * for each $\mathrm{H}$ tone that is not associated to the rightmost syllable of a NonMin foot.

8. MAX-LinK, DEP-LINK 
The combination of top-ranked tone faithfulness constraints and high-ranking LICENSE-H ensures the construction of a foot on top of the sponsor TBU. The foot directionality constraint, CHAIN-L $\left(\sigma_{\omega}\right)$, is decisive in determing the exact placement of the foot. High-ranking LICENSE-FT ensures that feet are only placed to license tones, and not to parse pairs of toneless syllables. The structural constraint *H/MinFT-L drives delinking from the left edge of feet, in effect causing tone to shift away from its underlying position. The tone licensing constraints LICENSE(H,MINFT-R) and LICENSE(H,NONMIN-R) designate the spreading targets for tone. The binary tonal span of the Saghala default pattern is thus the result of two separate spreading targets. Finally, low-ranking faithfulness constraints on tonal association links allow the grammar to freely delink and link tones if that is conducive to satisfying any markedness constraint.

Some of the rankings given above are not essential for the derivation of the default pattern. Specifically, the ranking between LICENSE-H and LICENSE-FT and the ranking among the bottom three markedness constraints is not crucial.

The next section will show how the given constraint ranking allows for a derivation of the Saghala default pattern.

4.1.1 Derivation The derivation follows the steps in (2). The derivation starts from an underlying fivesyllable form with tone on the second syllable: $/ \sigma \delta \sigma \sigma \sigma /$. From a five-syllable form it can be seen that the algorithm is not dependent on the proximity of a tone to a word edge. Given the underlying form, the desired surface form is tone only on the third and fourth syllables: [ $\sigma \sigma \sigma \sigma \sigma \sigma]$, which is indeed the output of the derivation, modulo foot structure.

\section{0. $\quad \sigma \sigma \sigma \sigma \sigma \sigma$}

Underlying Form

1. $\sigma(\dot{\sigma} \sigma) \sigma \sigma$

Foot placement.

2. $\sigma((\dot{\sigma} \sigma) \sigma) \sigma$

Rightward foot expansion.

3. $\sigma\left(\left(\dot{\sigma}^{\prime}\right) \sigma\right) \sigma$

Spreading to the right edge of MinFt.

4. $\sigma((\sigma \dot{\sigma}) \sigma) \sigma$

Delinking from the left edge of MinFt.

5. $\sigma((\sigma \dot{\sigma}) \dot{\sigma}) \sigma$

Spreading to the right edge of NonMinFt.

6. $\sigma\left(\left(\sigma \sigma^{\prime}\right) \dot{\sigma}\right) \sigma$

Termination of the HS algorithm; this is the output form.

The following tableaux will show each of the steps in detail. The top-ranked and bottom-ranked faithfulness constraints are left out of the tableaux. Candidates involving deletion or insertion of tones will not be considered. Consequently, all candidates contain exactly one tone, which may surface on multiple TBUs through multiple association. In all tableaux, the first candidate corresponds to a faithful mapping from the input form.

Firstly, tableau 3 shows the first step taken from the underlying form. Because of high-ranking LICENSE$\mathrm{H}$, it is optimal to construct a foot in such a way that it contains the sponsor TBU. The decision between having the sponsor at the left or right edge of the foot is left to CHAIN-L, which prefers having feet pulled rightward. Consequently, candidate $3 \mathrm{~b}$ is optimal, since it incurs less violations of CHAIN-L than 3c. Candidate $3 \mathrm{~d}$ is ideal in terms of CHAIN-L, but fails to satisfy the high-ranking licensing constraints, because it leaves the $\mathrm{H}$ tone unlicensed, and fails to license its newly created foot with a tone.

In tableau 4, the faithful mapping fails because of too many violations of CHAIN-L. Candidate $4 \mathrm{~b}$ shows the winning move, which is to expand the foot rightward, to parse an additional syllable. Candidate $4 \mathrm{c}$ shows that expanding instead to the left is not beneficial, because it is not in line with the orientation of CHAIN-L. Candidate $4 \mathrm{~d}$ shows the optimal satisfaction of CHAIN-L, constructing another binary foot. However, this comes at the cost of violating higher-ranked LICENSE-FT, and so the candidate is out. Finally, candidate 4e demonstrates that spreading is not a priority at this stage. 


\begin{tabular}{|c|c|c|c|c|c|c|}
\hline$\sigma \dot{\sigma} \sigma \sigma \sigma$ & $\mathrm{LICH}$ & LiCFT & CHAIN-L & *H/MIN-L & $\mathrm{R}(\mathrm{H}, \mathrm{MIN})$ & $\mathrm{R}(\mathrm{H}, \mathrm{NONMIN})$ \\
\hline a. $\quad \sigma \dot{\sigma} \sigma \sigma \sigma$ & $* !$ & & & & $*$ & $*$ \\
\hline b. $\sigma(\sigma \sigma) \sigma \sigma$ & & & $* *$ & $*$ & $*$ & $*$ \\
\hline c. $\quad(\sigma \dot{\sigma}) \sigma \sigma \sigma$ & & & $* * *$ & & & $*$ \\
\hline d. $\quad \sigma \dot{\sigma} \sigma(\sigma \sigma)$ & $* !$ & $*$ & & & $*$ & $*$ \\
\hline
\end{tabular}

Tableau 3: Default context, step 1.

\begin{tabular}{|l||c|c|c|c|c|c|}
\hline$\sigma(\sigma \sigma) \sigma \sigma$ & LICH & LICFT & CHAIN-L & $*$ H/MIN-L & R(H,MIN) & R(H,NONMIN) \\
\hline a. $\sigma(\sigma \sigma) \sigma \sigma$ & & & $* * !$ & $*$ & $*$ & $*$ \\
\hline b. $\sigma((\sigma \sigma) \sigma) \sigma$ & & & $*$ & $*$ & $*$ & $*$ \\
\hline c. $\quad(\sigma(\sigma \sigma)) \sigma \sigma$ & & & $* * !$ & $*$ & $*$ & $*$ \\
\hline d. $\sigma(\sigma \sigma)(\sigma \sigma)$ & & $* !$ & & $*$ & $*$ & $*$ \\
\hline e. $\quad \sigma(\sigma o ́) \sigma \sigma$ & & & $* * !$ & $*$ & & $*$ \\
\hline
\end{tabular}

Tableau 4: Default context, step 2.

After the second step, optimal parsing has been achieved, and the derivation turns to tone spreading. The faithful candidate 5a does not satisfy the lower-ranking tone licensing constraints, and so 5b, showing the result of a spreading operation, is preferable. Candidate 5c shows the result of spreading leftward: although it does not exacerbate the violations of any of the constraints shown, it does nothing to improve on the faithful candidate and so it is suboptimal. Note that delinking is premature at this point, because this would result in a floating tone, which is blocked by top-ranked NoFLOAT (not shown in tableau).

\begin{tabular}{|c|c|c|c|c|c|c|}
\hline$\sigma\left(\left(\sigma^{\prime} \sigma\right) \sigma\right) \sigma$ & LICH & LICFT & CHAIN-L & $* \mathrm{H} / \mathrm{MIN}-\mathrm{L}$ & $\mathrm{R}(\mathrm{H}, \mathrm{MIN})$ & $\mathrm{R}(\mathrm{H}, \mathrm{NONMIN})$ \\
\hline a. $\quad \sigma((\dot{\sigma} \sigma) \sigma) \sigma$ & & & $*$ & $*$ & $* !$ & $*$ \\
\hline b. $\sigma\left(\left(\sigma^{\prime} \sigma\right) \sigma\right) \sigma$ & & & $*$ & $*$ & & $*$ \\
\hline c. $\quad \dot{\sigma}((\dot{\sigma} \sigma) \sigma) \sigma$ & & & * & $*$ & $* !$ & $*$ \\
\hline
\end{tabular}

Tableau 5: Default context, step 3.

Since tone has now spread to a second TBU, it is possible to delink from the first TBU without creating a floating tone. This is shown in tableau 6 by candidate $6 \mathrm{~b}$, and it is the optimal move because *H/MIN-L outranks the tone licensing constraints.

After tone delinking, the time is ripe for the tone to associate to the second spreading target - the right edge of the non-minimal foot. This is achieved in tableau 7 by candidate $7 \mathrm{~b}$.

After reaching both its spreading targets and delinking from the sponsor, the derivation is complete. Tableau 8 shows the convergence of the algorithm as the faithful mapping is the optimal candidate. Candidate $8 \mathrm{~b}$ shows that further delinking is unwarranted, as it causes tone to no longer be licensed by the rightmost syllable of a minimal foot.

After Tableau 8, the derivation is finished. The output is $\sigma((\sigma \sigma) \hat{\sigma}) \sigma$, with surface tone at the two syllables following the sponsor, as desired. 


\begin{tabular}{|l||l|c|c|c|c|c|}
\hline$\sigma\left(\left(\sigma^{\prime}\right) \sigma\right) \sigma$ & LICH & LICFT & CHAIN-L & $*$ H/Min-L & R(H,Min) & R(H,NONMIN) \\
\hline a. $\sigma\left(\left(\sigma^{\prime}\right) \sigma\right) \sigma$ & & & $*$ & $* !$ & & $*$ \\
\hline b. $\sigma((\sigma \dot{\sigma}) \sigma) \sigma$ & & & $*$ & & & $*$ \\
\hline c. $\sigma\left(\left(\sigma^{\prime}\right) \dot{\sigma}\right) \sigma$ & & & $*$ & $* !$ & & \\
\hline
\end{tabular}

Tableau 6: Default context, step 4.

\begin{tabular}{|l||l|l|c|c|c|c|}
\hline$\sigma((\sigma \dot{\sigma}) \sigma) \sigma$ & LICH & LICFT & CHAIN-L & $*$ H/MIN-L & R(H,MIN) & R(H,NONMIN) \\
\hline a. $\sigma((\sigma \sigma) \sigma) \sigma$ & & & $*$ & & & $* !$ \\
\hline b. $\sigma((\sigma \dot{\prime}) \dot{\sigma}) \sigma$ & & & $*$ & & & \\
\hline
\end{tabular}

Tableau 7: Default context, step 5.

\begin{tabular}{|l||c|c|c|c|c|c|}
\hline$\sigma((\sigma \dot{\sigma}) \dot{\sigma}) \sigma$ & LICH & LICFT & CHAIN-L & $*$ H/MIN-L & R(H,MIN) & R(H,NONMIN) \\
\hline a. $\sigma\left(\left(\sigma \sigma^{\prime}\right) \dot{\sigma}\right) \sigma$ & & & $*$ & & & \\
\hline b. $\sigma\left((\sigma \sigma) \sigma^{\prime}\right) \sigma$ & & & $*$ & & $* !$ & \\
\hline
\end{tabular}

Tableau 8: Default context, step 6. 


\section{Conclusion}

This talk has introduced a renewed metrical approach to bounded tone in Bantu. Key elements are the adoption of a layered foot, the use of Harmonic Serialism to derive local effects, and the proposal of a licensing/structure constraint family to relate tone and feet to each other.

It was demonstrated that the framework can account for the default pattern of Saghala, which involves both tone shift and tone spread in a trisyllabic domain.

Future work will take a typological perspective, testing whether the proposed approach can apply to a wider range of attested patterns, and how a learnability perspective on the proposed analyses may help to fit the attested typology.

\section{References}

Bickmore, Lee S (1996). Bantu tone spreading and displacement as alignment and minimal misalignment .

Bickmore, Lee S \& Nancy C Kula (2013). Ternary spreading and the OCP in Copperbelt Bemba. Studies in African Linguistics 42:2.

Breteler, Jeroen (forthcoming). Metrical tone shift and spread in Harmonic Serialism: the case of Saghala.

Cassimjee, Farida \& Charles Kisseberth (1998). Optimal domains theory and bantu tonology. Kisseberth, Charles \& Larry Hyman (eds.), Theoretical aspects of Bantu tone, CSLI, 33-132.

Clements, George N (1984). Binding domains in Kikuyu. Studies in the Linguistic Sciences 14:2.

Cole, Jennifer \& Charles Kisseberth (1994). An optimal domains theory of harmony. Studies in the Linguistic Sciences $24: 2,1-13$.

Inkelas, Sharon \& Cheryl Zoll (2007). Is grammar dependence real? A comparison between cophonological and indexed constraint approaches to morphologically conditioned phonology.

Kager, René (1994). Ternary rhythm in alignment theory.

Kager, René (1999). Optimality Theory. Cambridge University Press, Cambridge.

Key, Michael (2007). Headed spans and Bantu tonology 1 .

Key, Michael \& Lee Bickmore (2014). Headed tone spans: binarity and minimal overlap. Southern African Linguistics and Applied Language Studies 32:1, 35-53.

Kiparsky, Paul (2000). Opacity and cyclicity. Linguistic review 17:2-4, 351-365.

Kisseberth, Charles W (1993). Optimal domains: A theory of Bantu tone (a case study from Isixhosa)'. Rutgers Optimality Workshop I.

Lakoff, George (1993). Cognitive phonology. Goldsmith, John (ed.), The last phonological rule: reflections on constraints and derivations, University of Chicago Press, 117-145.

Martínez-Paricio, Violeta (2013). An exploration of minimal and maximal metrical feet. Ph.D. thesis.

Martínez-Paricio, Violeta \& René Kager (forthcoming). Non-intervention constraints and the binary-to-ternary rhythmic continuum.

McCarthy, John J (2004). Headed spans and autosegmental spreading. Linguistics Department Faculty Publication Series 42.

McCarthy, John J (2010). An introduction to Harmonic Serialism. Language and Linguistics Compass 4:10, 1001-1018.

McCarthy, John J, Kevin Mullin \& Brian W Smith (2012). Implications of Harmonic Serialism for lexical tone association. Botma, Bert \& Roland Noske (eds.), Phonological Explorations. Empirical, Theoretical and Diachronic Issues, De Gruyter, 265-297.

Odden, David (2001). Tone shift and spread in Taita I. Studies in African Linguistics 30:1, p. 1.

Patin, Cédric (2002). Aspects du système accentuel du Saghala (dialecte Teri). Master's thesis, Université SorbonneNouvelle.

Patin, Cédric (2009). Tone shift and tone spread in the Saghala noun phrase. Faits de langues :1.

Prince, Alan S (1980). A metrical theory for Estonian quantity. Linguistic Inquiry 511-562.

Prince, Alan \& Paul Smolensky (1993). Optimality Theory: Constraint interaction in generative grammar .

Selkirk, Elisabeth O (1980). The role of prosodic categories in English word stress. Linguistic inquiry 563-605.

Sietsema, Brian Mark (1989). Metrical dependencies in tone assignment. Ph.D. thesis, Massachusetts Institute of Technology. 